\title{
Management of the Low-Flow Head and Neck Vascular Malformations in Children: the Sclerotherapy Protocol
}

\author{
Michael Leung ${ }^{1}$ Ling Leung ${ }^{1}$ Dickson Fung ${ }^{2}$ Wai-lun Poon ${ }^{2}$ Clarence Liu ${ }^{1}$ Kenneth Chung ${ }^{1}$ \\ Paula Tang ${ }^{1}$ Sunny Tse ${ }^{2}$ Tsz-wo Fan ${ }^{2}$ Nicholas Chao ${ }^{1}$ Kelvin Liu ${ }^{1}$
} ${ }^{1}$ Division of Pediatric Surgery, Department of Surgery, Queen
Elizabeth Hospital, Kowloon, Hong Kong
${ }^{2}$ Department of Radiology and Imaging, Queen Elizabeth Hospital,
Kowloon, Hong Kong
Address for correspondence Michael Leung, MBChB, FRCSEd, Division of Pediatric Surgery, Department of Surgery, Queen Elizabeth Hospital, 30 Gascoigne Road, Kowloon, Hong Kong (e-mail: leungwym@ha.org.hk).

Eur J Pediatr Surg 2014;24:97-101.

\begin{abstract}
Keywords

- vascular malformations

- head and neck

- sclerotherapy

Aim Image-guided sclerotherapy is becoming the preferred treatment for low-flow vascular malformations in head and neck region. The authors review the management protocol for this condition and evaluate its clinical outcomes.

Methods Children with low-flow vascular malformations in head and neck region undergoing sclerotherapy from 2010 to 2013 were reviewed. All patients were assessed by pediatric surgeons and interventional radiologists in the multidisciplinary vascular anomalies clinic. Ultrasonography and intravenous contrast enhanced magnetic resonance imaging were performed preoperatively. Under general anesthesia with endotracheal intubation, sclerotherapy were performed with ultrasonographic and fluoroscopic guidance. Sodium tetradecryl sulfate (STS) foam or ethanolamine was used for venous malformation and doxycycline for lymphatic malformations as primary sclerosants, whereas $98 \%$ ethanol was reserved as an adjuvant sclerosant in selected cases of repeated procedures. Perioperative dexamethasone $0.2 \mathrm{mg} / \mathrm{kg}$ thrice daily was administered to decrease postsclerotherapy swelling and single dose intravenous mannitol $0.5 \mathrm{~g} / \mathrm{kg}$ was given to minimize thromboembolic complications. Postoperatively, patients were admitted to intensive care unit for mechanical ventilation under deep sedation for airway protection.

Results Overall 13 children ( 8 male and 5 female) with a mean age of 25 months (range, 2 mo-11 y) underwent a total of 25 sessions of image-guided staged sclerotherapy. There were five venous and eight lymphatic malformations. Location wise there were eight cervical, one lingual, one parotid, one lip, one facial, and one palatal lesions. Six patients had obstructive airway symptoms. Five patients required staged sclerotherapies from two to six sessions. There were no airway and thromboembolic complications. One patient had bleeding while another had recurrent swelling following sclerotherapy for lymphatic malformations and they were treated by aspiration. Significant size reductions of more than $50 \%$ volume were achieved in all patients. All patients with obstructive symptoms showed improvement.

Conclusion Sclerotherapy is a safe and effective treatment for head and neck vascular malformations in children. Routine perioperative protocol is essential to reduce airway
\end{abstract}

received

May 14, 2013

accepted after revision

July 18, 2013

published online

September 5, 2013 (c) 2014 Georg Thieme Verlag KG

Stuttgart · New York
DOI http://dx.doi.org/ 10.1055/s-0033-1354585. ISSN 0939-7248. 
and thromboembolic complications. Size reduction and functional improvement occurred in all patients undergoing sclerotherapy.

\section{Introduction}

Mulliken and Glowacki proposed the classification of vascular anomalies in 1982. Based on clinical course and histologic characteristics, this classification divides vascular anomalies into tumors and malformations. ${ }^{1}$ Vascular malformations are subdivided depending on (1) the predominant type of vessels involved (capillary, venous, arteriovenous, lymphatic, or combined) and (2) the flow characteristics (low-flow, highflow, and combined lesions). ${ }^{2,3}$

Venous and lymphatic malformations are low-flow vascular malformations. Majority of them occur in the head and neck region and can infiltrate normal structures. They can give rise to functional disabilities, including obstruction to airway and swallowing difficulties. Also, they can cause visual or auditory disturbance and delayed speech development. In addition, bleeding, pain, infection, skeletal deformity, and cosmetic disfigurement may occur.

Clinical diagnosis is made by taking history details and physical examination. Radiological investigations aim for confirming the diagnosis and define the anatomy of lesion in relation to the surrounding structures, which are important for planning of sclerotherapy. Ultrasonography (USG) with Doppler scan and intravenous contrast enhanced magnetic resonance imaging (MRI) are commonly performed.

Unlike most hemangiomas that will involute before puberty, venous malformations will grow with age, infiltrating normal structures. Most lymphatic malformations persist and can be acutely enlarged because of bleeding and inflammation. Thus, active treatment of venous and lymphatic malformations is indicated especially in head and neck region. Surgery can cause damage to normal surrounding structures, resulting in significant disfigurement and loss of function. Complete excision is often impossible for infiltrative lesions. Image-guided sclerotherapy has gained popularity recently and become the first-line treatment for low-flow vascular malformations in head and neck region. The authors review the management protocol for this condition and evaluate the clinical outcomes. ${ }^{4-7}$

\section{Material and Methods}

A retrospective review of all children below age 18 years with vascular malformations undergoing image-guided sclerotherapy from 2010 to 2013 was done. Patients with low-flow vascular malformations involving the head and neck region were included. Patient demographics, presenting symptoms, clinical and radiological findings were collected. All patients were assessed by pediatric surgeons and interventional radiologists in the multidisciplinary vascular anomalies clinic. USG and intravenous contrast enhanced MRI were performed preoperatively. Platelet count, clotting profile, and serum D-dimer level was checked before sclerotherapy.
For large vascular malformations with potential airway involvement, the authors would plan for staged sclerotherapy to control the postsclerotherapy edema and prevent sclerosant overdose. Sclerotherapy was performed under general anesthesia. On induction, intravenous mannitol $0.5 \mathrm{~g} / \mathrm{kg}$ was infused to expand the intravascular volume, preventing thromboembolic complications. A Foley catheter was inserted to monitor urine output and detect hematuria. Endotracheal intubation was performed in all patients for airway protection. Intravenous dexamethasone $0.2 \mathrm{mg} / \mathrm{kg}$ together with esomeprazole was given intraoperatively to control postsclerotherapy edema.

Sclerotherapy was performed with sonographic and fluoroscopic guidance. A 24-gauge angiocatheter was inserted to the lesion under USG guidance. Presclerotherapy fluoroscopy was performed by injecting 50\% diluted Omnipaque 300 (GE HealthCare Inc., Wauwatosa, Wisconsin, United States) in normal saline to the lesion to define the anatomy of vascular malformation. Also, it could minimize extravasation and spillage to sclerosant during sclerotherapy.

Different types of sclerosants were used in the authors' series. STS foam or ethanolamine was used as first-line sclerosant for venous malformations. STS foam was produced by mixing $2 \mathrm{~mL} 3 \% \mathrm{STS}, 0.5 \mathrm{~mL}$ Lipiodol (Guerbet Group, Villepinte, France) and $2.5 \mathrm{~mL}$ air. Doxycycline was the primary sclerosant for lymphatic malformations. For resistant lesions, $98 \%$ ethanol was added as adjuvant sclerosant in selected cases of repeated sclerotherapies. Sclerosant was deployed to the lesion under fluoroscopic guidance. In venous malformation of lip, tongue, and palate, vascular (CooleyDerra or Debakey) clamp was applied to control the venous outflow and compartmentalize the injected sclerosant.

If the vascular malformations had potential airway involvement, the patients would be admitted to intensive care unit after sclerotherapy. They were deeply sedated with airway protected by endotracheal intubation and mechanical ventilation. Intravenous dexamethasone was continued 8 hourly for few days in decreasing dose according to the extent of postsclerotherapy edema. Patients were closely observed and extubated when the edema of lesion subsided. The patients would be transferred to general ward after uneventful extubation. If they tolerated diet well and showed no postoperative complications, they would be discharged home in 1 or 2 days. After discharge, they were followed up in the multidisciplinary vascular anomalies clinic at 2 weeks, 3, 6, and 12 months. More frequent follow-up was offered if the patients had persistent or recurrent lesion needing repeated sclerotherapies. USG was performed 6 months after sclerotherapy to assess the size of malformations. MRI was reserved for those patients with persistent lesions planned for repeated sclerotherapies.

The primary outcome measure was the size of vascular malformations after treatment. Major size reduction was defined as more than $50 \%$ decrease in size of lesions. 
Secondary outcome measures included the perioperative complications, status of obstruction symptoms, and cosmetic results.

Obstruction was defined as swallowing symptoms including slow feeding, drooling of saliva and choking; or upper airway symptoms such as stridor, noisy breathing, snoring, and obstructive sleep apnoea.

\section{Results}

Overall 40 patients (21 girls and 19 boys) had low-flow vascular malformations with sclerotherapy performed in the study period. Among them, 27 patients had vascular malformations at trunk or limbs. Thirteen children ( 8 males and 5 females) had involvement in head and neck region and they were recruited in this study. There were five venous and eight lymphatic malformations. The sites of vascular malformations included eight cervical (62\%), one lingual (8\%), one parotid (8\%), one lip (8\%), one facial (8\%), and one palatal (8\%) lesions. The mean age at treatment was 25 months (range, 2 mo-11 y).

Overall 10 patients (77\%) presented with a mass or swelling, 2 patients (15\%) with pain after bleeding, 1 patient (8\%) with bluish discoloration of the overlying skin, 6 patients (46\%) had obstructive upper airway symptoms, and 1 patient (8\%) had feeding difficulty.

All patients underwent sclerotherapy with a total of 25 sessions of sclerotherapy done. Eight patients underwent one sclerotherapy session, two patients underwent two sclerotherapy sessions. One patient underwent three sessions. He suffered from a $5 \mathrm{~cm}$ diameter left parotid venous malformation with stridor and sleep apnoea at the age of 3 years. Sclerotherapy sessions were performed at 5 and 9 months interval. One patient had four treatment sessions. She was born with congenital giant lymphatic malformation involving left side of face extending into the pharyngeal spaces and submandibular area across the midline. She had upper airway obstruction with tracheostomy performed. Multistaged sclerotherapy was performed. One patient was born with congenital giant lymphatic malformation involving right neck, extending through the right axilla, chest, and lower abdomen. He underwent partial excision of the lesion, followed by five percutaneous OK-432 sclerotherapy sessions in other centers. Doxycycline sclerotherapy was performed at 6 years of age.

The median duration of follow-up was 1.5 years (range, 2 mo-3 y). Clinical significant size reductions of more than $50 \%$ volume were achieved in all patients. From postoperative imaging, complete resolution occurred in two patients (15\%). All patients with obstructive symptoms showed improvement.

There were no airway complications. One patient had mild hematuria after STS foam sclerotherapy for venous malformation. The condition subsided after intravenous fluid supplement. One patient with lymphatic malformation had postsclerotherapy bleeding and one had recurrent swelling. USG-guided aspiration of lymphatic cysts was done.

\section{Discussion}

Vascular anomalies are broadly classified into vascular tumors and vascular malformations. Infantile hemangioma is the most common vascular tumor characterized by an initial proliferative phase followed by involution after 1 year of age. It is responsive to propranolol and interventional treatment is often not required. ${ }^{8}$ In contrast, vascular malformations do not involute and will persist. Bleeding or infection of cysts could occur in lymphatic malformations, causing acute swelling and pain. Enhanced infiltrative growth of venous malformations occur under hormonal influence especially in puberty, steroid therapy, or pregnancy. ${ }^{9}$ They can cause worsening symptoms with detrimental functional complications. Intervention should be considered before adolescence.

Vascular malformations can be further differentiated according to the flow characteristic and the type of vessel involved. The classification according to the hemodynamic properties is of important clinical significance. High-flow vascular malformations, such as arteriovenous fistulas and arteriovenous malformations, are treated by transarterial embolization, whereas low-flow malformations can be treated with sclerotherapy. ${ }^{10}$

In this study, the authors focus on low-flow vascular malformations causing mechanical symptoms to head and neck region. Venous malformations and lymphatic malformations are the most common conditions. Venous malformations account for approximately $60 \%$ of low-flow vascular malformations. A bluish discoloration of the overlying skin or mucosa may occur. Doppler ultrasound will demonstrate a heterogeneous spongy-like lesion with tortuous vessels. Other sonographic characteristics are expansion during valsalva maneuvers, phleboliths, and absence of dilated feeding arteries and draining veins. ${ }^{1,4,11-14}$ Lymphatic malformations can be subclassified into macrocystic and microcystic, which is of therapeutic importance. Macrocystic lymphatic malformations can be treated effectively by sclerotherapy. ${ }^{15}$ In microcystic lesions, treatment response to sclerotherapy is suboptimal and excision is often required. More than twothird of lymphatic malformations are found in the soft tissue of the neck and are often associated with inflammations and bleeding. ${ }^{11}$ USG appearance of macrocystic lymphatic malformations are homogenous and hypoechoic. After hemorrhage and infection, cysts with mixed echogenicity are revealed under USG.

The infiltrative and extensive nature of vascular malformations in the head and neck region often makes resection impossible without sacrificing the vital structures. Sclerotherapy, a minimal invasive treatment modality that involves injection of chemical agent to cause inflammation of vascular endothelium, has become the first-line treatment. ${ }^{4,5,16}$

Vascular malformations in head and neck region are exceptionally challenging clinical conditions that require multidisciplinary management. Commonly used sclerosants induce endothelium inflammation and edema of surrounding tissue. In head and neck region they can cause airway obstruction postoperatively. Extravasation of sclerosants can cause skin or muscle necrosis and neuropathy. Spillage 
of excessive sclerosants to cerebral and pulmonary circulations may result in detrimental complications such as stroke and pulmonary embolism. ${ }^{17}$ Thus, it is essential to have extra care when performing sclerotherapy for vascular malformations in head and neck region. In the authors' series all patients were assessed in the multidisciplinary vascular anomalies clinic preoperatively, with participation of pediatric surgeons, interventional radiologists, anesthesiologists, pediatric intensivists, and nurse specialists. USG and intravenous contrast enhanced MRI were performed. Before sclerotherapy, thrombocytopenia and deranged clotting profile were excluded. Interestingly, localized intravascular coagulopathy is common in venous malformations with increased serum D-dimer level. ${ }^{18}$ Depending on the volume and sites of vascular malformations, excessive sclerosant injection may cause uncontrolled edema affecting airway or overdose toxicity. Five patients in the authors' series had staged procedures. Repeated sclerotherapy is considered not as a failure but rather a necessary strategy in management of large vascular malformations.

Several maneuvers are performed to control the postsclerotherapy edema. Intravenous dexamethasone was given intraoperatively and continued for a few days after the procedure. In every patient, sclerotherapy was done under general anesthesia with endotracheal intubation. The endotracheal tube was kept after procedure and patient was transferred to intensive care unit for mechanical ventilation if there was concern for postsclerotherapy airway edema.

Systemic spillage and extravasation of sclerosants have been reported. They can lead to skin necrosis, neuropathy, muscle atrophy and contracture, deep vein thrombosis, pulmonary embolism, disseminated intravascular coagulation, and cardiopulmonary collapse. ${ }^{19,20}$ Siniluoto et al reported one major complication of blindness following injection into an ophthalmic vein. ${ }^{21}$ Berenguer et al reported two transient facial weakness and one permanent unilateral vocal cord paralysis. ${ }^{10}$ In the study by Lee, eight patients developed deep venous thromboses and nine patients developed peripheral nerve damage, in which two were permanent. ${ }^{5}$ Birmingham study reported superficial skin necrosis that resolved conservatively. ${ }^{22}$ To minimize these complications, all the authors' patients had fluoroscopy performed before sclerotherapy by injecting water-soluble contrast to the targeted vascular malformations. Intravenous mannitol was given to expand the intravascular volume to prevent thromboembolism. Hemoglobinuria may occur and all their patients had Foley catheter inserted during sclerotherapy. One patient in the authors' series developed transient hematuria postoperatively. It was treated conservatively by rehydration. In three patients with venous malformations at lip, tongue and palate, the authors used vascular clamps were used to occlude the venous outflow and prevent sclerosant spillage to ophthalmic vein and cavernous sinus.

Although only two patients in the authors' series had complete radiological resolution of lesion, clinical significant volume reduction, and symptoms improvement occurred in all patients. The principle of sclerotherapy treatment is to control the lesion by size reduction. Eradication of vascular malformations is not possible in all modalities of treatment including surgical resection.

No airway and thromboembolic complications were observed in this series. The authors' treatment protocol aims to prevent airway and thromboembolic complications in the management of vascular malformations of the head and neck in children. Comparing with other series in the literature, their result showed an excellent response rate and the complication rate is low, all being minor and transient. In the series from Tan et $\mathrm{al}^{23} 70 \%$ achieved a positive clinical response and there were no major complications. There was a $75 \%$ positive clinical response rate observed by Berenguer et al. However, there were 42 complications occurred and 14 of those were major. ${ }^{10}$ Siniluoto et al reported a success rate of $82 \%$ and there was one major complication of blindness. ${ }^{21}$ Lee et al reported a $99 \%$ success rate and a complication rate of $51 \%{ }^{5}$ In the Birmingham study, $84 \%$ of patients showed improvement, but $18 \%$ had complication, with one being major. $^{22}$

One limitation in this retrospective study is the lack of standardization of sclerosants used. The choice of sclerosants was dependent on the supply and preference of interventional radiologists. Also, the outcomes measurement may be biased as the observers may have involved in the treatment protocol. A prospective study with independent observers blinded to the treatment protocol is necessary to evaluate the long-term results in future.

\section{Conclusion}

Sclerotherapy is a safe and effective treatment for head and neck vascular malformations in children. Routine perioperative protocol is essential to reduce airway and thromboembolic complications. Size reduction and functional improvement occurred in all patients undergoing sclerotherapy.

\section{Conflict of Interest}

None.

\section{References}

1 Mulliken JB, Glowacki J. Hemangiomas and vascular malformations in infants and children: a classification based on endothelial characteristics. Plast Reconstr Surg 1982;69(3):412-422

2 Ethunandan M, Mellor TK. Haemangiomas and vascular malformations of the maxillofacial region-a review. Br J Oral Maxillofac Surg 2006;44(4):263-272

3 Jackson IT, Carreño R, Potparic Z, Hussain K. Hemangiomas, vascular malformations, and lymphovenous malformations: classification and methods of treatment. Plast Reconstr Surg 1993;91 (7):1216-1230

4 Puig S, Casati B, Staudenherz A, Paya K. Vascular low-flow malformations in children: current concepts for classification, diagnosis and therapy. Eur J Radiol 2005;53(1):35-45

5 Lee BB. New approaches to the treatment of congenital vascular malformations (CVMs)-a single centre experience. Eur J Vasc Endovasc Surg 2005;30(2):184-197 
6 Kane WJ, Morris S, Jackson IT, Woods JE. Significant hemangiomas and vascular malformations of the head and neck: clinical management and treatment outcomes. Ann Plast Surg 1995;35(2): 133-143

7 Greene AK, Burrows PE, Smith L, Mulliken JB. Periorbital lymphatic malformation: clinical course and management in 42 patients. Plast Reconstr Surg 2005;115(1):22-30

8 Léauté-Labrèze C, Dumas de la Roque E, Hubiche T, Boralevi F, Thambo JB, Taïeb A. Propranolol for severe hemangiomas of infancy. N Engl J Med 2008;358(24):2649-2651

9 Hyder SM, Huang JC, Nawaz Z, et al. Regulation of vascular endothelial growth factor expression by estrogens and progestins. Environ Health Perspect 2000;108(Suppl 5):785-790

10 Berenguer B, Burrows PE, Zurakowski D, Mulliken JB. Sclerotherapy of craniofacial venous malformations: complications and results. Plast Reconstr Surg 1999;104(1):1-11, discussion 12-15

11 Werner JA, Eivazi B, Folz BJ, Dünne AA. State of the art of classification, diagnostics and therapy for cervicofacial hemangiomas and vascular malformations [in German]. Laryngorhinootologie 2006;85(12):883-891

12 Eivazi B, Ardelean M, Bäumler W, et al. Update on hemangiomas and vascular malformations of the head and neck. Eur Arch Otorhinolaryngol 2009;266(2):187-197

13 Meyer JS, Hoffer FA, Barnes PD, Mulliken JB. Biological classification of soft-tissue vascular anomalies: MR correlation. AJR Am J Roentgenol 1991;157(3):559-564

14 Abernethy LJ. Classification and imaging of vascular malformations in children. Eur Radiol 2003;13(11):2483-2497
15 Wiegand S, Eivazi B, Zimmermann AP, Sesterhenn AM, Werner JA. Sclerotherapy of lymphangiomas of the head and neck. Head Neck 2011;33(11):1649-1655

16 Garzon MC, Huang JT, Enjolras O, Frieden IJ. Vascular malformations: Part I. J Am Acad Dermatol 2007;56(3):353-370, quiz 371-374

17 Cavezzi A, Parsi K. Complications of foam sclerotherapy. Phlebology 2012;27(Suppl 1):46-51

18 Dompmartin A, Ballieux F, Thibon P, et al. Elevated D-dimer level in the differential diagnosis of venous malformations. Arch Dermatol 2009;145(11):1239-1244

19 Yakes WF, Haas DK, Parker SH, et al. Symptomatic vascular malformations: ethanol embolotherapy. Radiology 1989;170 (3 Pt 2):1059-1066

20 Takayasu K, Mizuguchi Y, Muramatsu Y, et al. Late complication of a large simple cyst of the liver mimicking cystadenocarcinoma after sclerotherapy. AJR Am J Roentgenol 2003;181(2):464-466

21 Siniluoto TM, Svendsen PA, Wikholm GM, Fogdestam I, Edström S. Percutaneous sclerotherapy of venous malformations of the head and neck using sodium tetradecyl sulphate (sotradecol). Scand J Plast Reconstr Surg Hand Surg 1997;31(2):145-150

22 Kok K, McCafferty I, Monaghan A, Nishikawa H. Percutaneous sclerotherapy of vascular malformations in children using sodium tetradecyl sulphate: the Birmingham experience. J Plast Reconstr Aesthet Surg 2012;65(11):1451-1460

23 Tan KT, Kirby J, Rajan DK, et al. Percutaneous sodium tetradecyl sulfate sclerotherapy for peripheral venous vascular malformations: a single-center experience. J Vasc Interv Radiol 2007;18 (3):343-351 